\title{
Endocardite Infecciosa em Adolescentes. Análise dos Fatores de Risco de Mortalidade Intra-Hospitalar
}

\author{
Nádia Barreto Tenório Aoun, Francisco Manes Albanesi F, Maurício Bastos de Freitas Rachid, \\ Luíz André Vieira Fernandes, José Augusto da Silva Messias \\ Rio de Janeiro, RJ
}

Objetivo - Estudar os aspectos epidemiológicos, clínicos, terapêuticos e evolutivos da endocardite infecciosa (EI) em grupo de pacientes com idade entre 12 e 20 (média de 15,5) anos.

Métodos - Foram estudados, retrospectivamente, 33 pacientes consecutivos (14 do sexo masculino e 19 do feminino), admitidos com diagnóstico de EI.

Resultados - A mortalidade da EI foi de $42 \%$. A cardiopatia reumática predominou como condição predisponente (63\% dos casos), seguida das cardiopatias congênitas (24\%) e próteses cardíacas (12\%). A maioria dos pacientes (78\%) encontrava-se na admissão em CF III e IV e apresentava mortalidade significativamente maior do que os que se encontravam na CF I e II ( $p=0,01)$. Complicações embólicas foram detectadas em $51 \%$ e determinaram maior mortalidade $(p=0,05)$. O agente etiológico mais isolado foi o Staphylococcus aureus (em $42 \%$ das hemoculturas positivas), seguido do Staphylococcus viridans (em 21\%). A análise multivariada mostrou que a contagem global de leucócitos acima de $10.000 / \mathrm{mm}^{3}$, e a CF referidos na admissão $(p=0,01$ e $p=0,04)$, e a ocorrência de embolias $(p=0,03)$ eram preditores independentes de mortalidade intra-hospitalar.

Conclusão - A cardiopatia reumática permanece, semelhante à população adulta, como principal fator predisponente da EI nos adolescentes, tendo como principal agente etiológico o S.aureus, semelhante à população pediátrica. A mortalidade é elevada e representam preditores de mortalidade intra-hospitalar a CF na admissão, a ocorrência da fenômenos embólicos e a leucocitose.

Palavras -chave: endocardite infecciosa, adolescente, cirurgia cardíaca

\section{Infective Endocarditis in Adolescents. Analy- sis of Risk Factors for In-Hospital Mortality}

Purpose - To study the epidemiological, clinical, therapeutic and evolutive aspects of endocarditis in a group of patients aging 12 to 20 years-old (mean 15.5).

Methods - Thirty-three consecutive patients (14 males, 19 females) admitted with infective endocarditis were retrospectively studied.

Results - Infective endocarditis mortality was $42 \%$. Rheumatic heart disease was the predominant underlying condition in $63 \%$ of patients. Congenital heart disease $(24 \%)$ and cardiac prosthesis $(12 \%)$ were the other affections involved. The majority of patients (78\%) were in functional class III and IV, with more deaths than the $22 \%$ who were in functional class I and II $(p=0.01)$. Staphylococcus aureus was the most frequently isolated agent (42\% of the positive blood cultures, followed by Staphylococcus viridans, 21\%). Multivariate analysis identified total leukocyte count above 10,000/ $\mathrm{mm}^{3}$ and functional class, both at admission $(p=0.01$ and $p=0.004$, respec tively), and the occurrence of embolic complications $(p=0.03)$ as independent predictors of in-hospital mortality.

Conclusion - Rheumatic heart disease remains, as in adults, the main predisposing factor for infective endocarditis in adolescents, and S.aureus is, like in children, the leading agent. Mortality is high and functional class at hospital admission, embolic complications and leukocytosis are independent predictors of in-hospital mortality.

Key-words: infective endocarditis, adolescent, cardiac surgery
Hospital Universitário Pedro Ernesto - UERJ

Correspondência: Nádia Barreto T. Aoun - Av. Maracanã, 617/603 - B1. 1 - 20511 000, Rio de Janeiro, RJ

Recebido para publicação em 24/7/97

Aceito em 16/10/97
A endocardite infecciosa (EI) é uma doença grave, responsável por uma a cada mil admissões hospitalares ${ }^{1,2}$. É ainda mais rara na infância, não ultrapassando nos Estado Unidos da América do Norte, 0,34 casos por 100.000 crianças ao ano ${ }^{3}$, representando um caso para cada 4.500 
internações, razão pela qual há poucos trabalhos sobre este tema na população pediátrica. Não existem estudos específicos sobre o comportamento desta doença na adolescência, sendo os diversos aspectos analisados conjuntamente com os da população adulta ou pediátrica ${ }^{4-8}$. O Núcleo de Estudo da Saúde do Adolescente (NESA) é um centro especializado no atendimento de adolescentes e, portanto, centro de referência estadual, o que nos permite observar número expressivo desta doença, que representa 1 a $2 \%$ do total de casos ao ano ou 10 a cada 1000 internações hospitalares. O objetivo deste trabalho é analisar os diversos aspectos relacionados à doença e os fatores de risco de mortalidade intra-hospitalar, em um grupo de pacientes adolescentes, com idade entre 12 e 20 anos.

\section{Métodos}

Foram revistos, retrospectivamente, 37 pacientes internados na enfermaria do NESA, com diagnóstico de EI, de março/94 a junho/85, sendo que quatro deles foram excluídos por não apresentarem dados suficientes para avaliação em seus prontuários médicos ou por não preencherem os critérios atuais de EI, propostos por Durack e col ${ }^{9}$. Dos 33 pacientes estudados, $19(57 \%)$ eram do sexo feminino e 14 (43\%), do masculino, com idades variando entre 12 e 20 (média 15,5) anos. Foram revistos: classe funcional (CF) segundo a New York Heart Association (NYHA), doença cardíaca de base, possível porta de entrada, presença de febre, ocorrência de complicações embólicas, resultados das hemoculturas, aspectos do hemograma, do eletrocardiograma, da telerradiografia de tórax, presença de vegetação ao ecocardiograma (ECO), tipo de tratamento empregado e a mortalidade da doença e, finalmente, a evolução desses pacientes, com o objetivo de apurar a ocorrência de novos episódios de EI, complicações tardias necessitando de correção cirúrgica, óbitos não relacionados à internação e a situação atual, quanto à $\mathrm{CF}$.

As comparações entre as variáveis contínuas foram realizadas com o teste t de Student, quando a distribuição era normal e, em caso contrário, utilizado o teste de MannWhitney. As comparações entre as variáveis categóricas foram realizadas pelos testes do qui-quadrado e de probabilidades exatas de Fisher, quando apropriados. Para determinação da normalidade das distribuições foi empregado o teste de goodness-of-fit. Um modelo multivariado de riscos proporcionais de Cox foi construído para identificar os preditores independentes de óbito intra-hospitalar, a partir de dados da admissão. As curvas de sobrevida atuarial foram elaboradas pelo método de Kaplan-Meier e comparadas, empregando-se o teste do log-rank. A regressão logística múltipla por etapas foi utilizada com o intuito de identificar os preditores independentes do óbito intra-hospitalar, a partir de dados obtidos na admissão e durante o período de internação. Foram considerados significativos os valores de $\mathrm{p}<0,05$.

\section{Resultados}

Quanto à cardiopatia de base, 21 (63\%) eram portadores de lesões orovalvares reumáticas (lesão mitral em 52\%, mitral e aórtica em 33\% e aórtica em 14\%.), 8 (24\%) de cardiopatias congênitas (comunicação interventricular (CIV) em 37\%) e 4 (12\%) de próteses cardíacas (sendo dois com próteses biológicas em posição mitral e dois, de próteses mecânicas em posição mitral e aórtica). Na internação, 17 (51,51\%) encontravam-se na CF IV , 9 $(27,27 \%)$ na CFIII, $5(15,15 \%)$ na CFII e $2(6,06 \%)$ na CFI. A distribuição quanto à idade, sexo e cardiopatia de base encontra-se na tabela I.

Em apenas 8 (24\%) dos 33 casos, foi identificada a possível porta de entrada: dois, devida à manipulação dentária sem profilaxia antibiótica, quatro, a lesões purulentas de pele por traumatismo (identificadas ao exame clínico), um, a parto vaginal há um mês, e um, a cirurgia cardíaca há oito dias. Não havia relato ou evidência de uso de drogas injetáveis e os pacientes que apresentavam vegetações no lado direito do coração eram portadores de lesões congênitas com comunicações intracardíacas.

Em relação aos exames complementares, a anemia foi achado freqüente; todos, exceto um (que apresentou EI no pós-operatório imediato de cirurgia cardíaca e submetido à hemotransfusão), apresentaram alterações nos valores do hematócrito (entre 14 e 33\%) e hemoglobina (entre 5 e $10 \mathrm{~g} \%)$. Em $13(39,39 \%)$ casos, a contagem global dos leucócitos encontrava-se acima de $10.000 / \mathrm{mm}^{3}$. As hemoculturas foram positivas em $21(63,63 \%)$ e negativas em 12 $(36,36 \%)$, sendo que, $5(41,66 \%)$ tinham se submetido a tratamento prévio com antibióticos. O Staphylococcus aureus foi isolado em 9 (42,85\%), seguido do Streptococcus, grupo viridans em $5(23,80 \%)$. Entre os portadores de endocardite

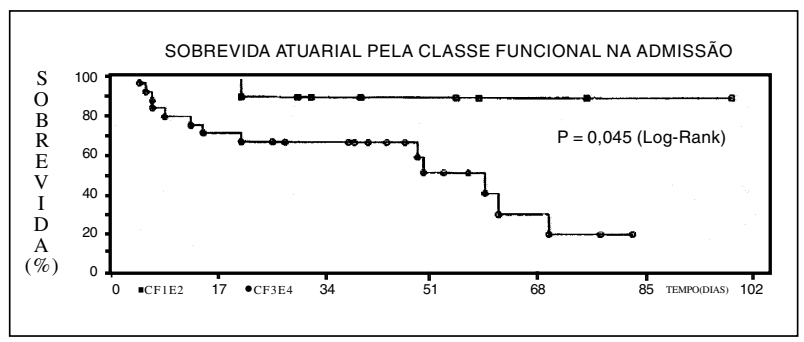

Fig. 1 - Sobrevida atuarial conforme a classe funcional na admissão. $\mathrm{CF}=$ classe funcional segundo classificação da "New York Heart Association"

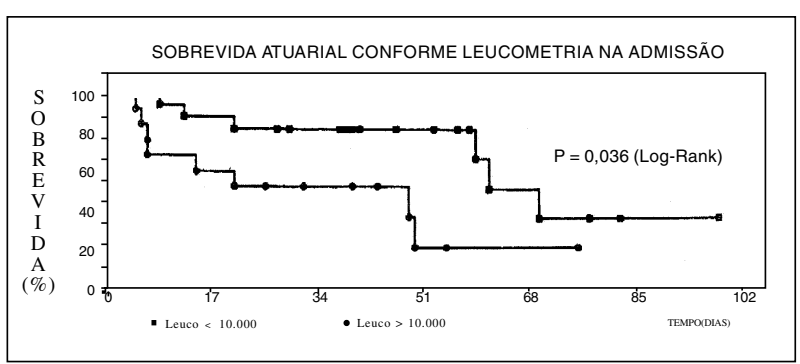

Fig. 2 - Sobrevida atuarial conforme leucometria na admissão. Leuco=leucometria global expressa em $\mathrm{mm}^{3}$ 


\begin{tabular}{|cccc|}
\hline \multicolumn{5}{|c|}{ Tabela I - Distribuição dos pacientes quanto à idade, sexo e cardiopatia } \\
de base na internação
\end{tabular}

estafilocócica, $8(88,88 \%)$ encontravam-se em CF III/IV, enquanto que os 12 com hemoculturas negativas, $10(83,33 \%)$ achavam-se em CF III/IV e nos 12 restantes, portadores de EI por outros agentes, $8(66,66 \%)$ encontravam-se em CF III/IV. Não houve diferença estatisticamente significativa entre o resultado das hemoculturas e a $\mathrm{CF}$ dos pacientes $(p>0,05)$. A relação entre os resultados das hemoculturas, a CF na internação e evolução dos pacientes encontram-se na tabela II.

Vegetações ao ECO foram detectadas em $25(75,75 \%)$ pacientes. A valva mitral foi acometida em 14 (56\%), sendo 12 valvas nativas e duas próteses biológicas, enquanto a valva aórtica esteve comprometida em nove (36\%) casos, sendo sete valvas nativas, uma prótese mecânica e um CIV subaórtico com acometimento da valva aórtica. Os dois restantes apresentavam vegetação nas valvas tricúspide (um) e pulmonar (um). Entre estes 25 pacientes, 20 (80\%) encontravam-se em CF III/IV e 5 (20\%), em CF III/IV. Dos oito em que não foram detectadas vegetações, seis (75\%) estavam em CF III/IV e dois (25\%), em CF III/IV. Não houve diferença estatística entre a presença de vegetação ao ECO e a CF ( $p>0,05)$, nem entre as valvas acometidas (aórtica ou mitral) ( $p>0,05)$. No entanto, todos os casos com envolvimento da valva mitral se achavam-se em CF III/IV, enquanto seis dos nove casos em que a valva aórtica esteve envolvida, encontravam-se em CFIII/IV ( $\mathrm{p}=0,04)$.
Embolias foram detectadas em 17 (51,51\%) dos 33 pacientes, sendo quatro oclusões arteriais agudas para membros inferiores, sete neurológicas, duas pulmonares e quatro cutâneas. Dos 25 casos com vegetação ao ECO, 15 (60\%) apresentaram embolias, enquanto $10(40 \%)$ não as apresentaram; e entre os casos sem vegetação, dois (25\%) tiveram complicações embólicas, enquanto seis (75\%) não as apre$\operatorname{sentaram}(\mathrm{p}>0,05)$.

Em relação ao tratamento instituído, 19 (57\%) foram submetidos a tratamento clínico exclusivo (14 (73\%) em CF III/IV e cinco ( $26 \%$ ) em CF I/II), sendo que nove ( $47 \%$ ) falecerame $10(52 \%)$ ficaram curados. Em 14(43\%) foramempregados os tratamentos clínico e cirúrgico $(12(85 \%)$ em CF III/ IV e dois (14\%) em CFI/II), sendo que cinco (35\%) faleceram e nove (64\%) obtiveram a cura. Não houve diferença estatística significativa entre os tratamentos empregados e a mortalidade neste estudo ( $p>0,05)$. As principais causas de óbito foram semelhantes nos dois tipos de tratamento: insuficiência cardíaca congestiva (quatro casos no clínico e três no cirúrgico), sepse (quatro no clínico e um no cirúrgico) e acidente vascular encefálico (uma rotura de aneurisma micótico no clínico e uma embolia cerebral no cirúrgico).

Em relação à mortalidade, observamos que a CF III/IV

\begin{tabular}{|c|c|c|c|}
\hline \multicolumn{4}{|c|}{$\begin{array}{l}\text { Tabela II - Resultados das hemoculturas, classe funcional na } \\
\text { internação e evolução dos pacientes }\end{array}$} \\
\hline Paciente & Hemocultura & $\mathrm{CF}$ & Evolução \\
\hline 1 & S. aureus & IV & Óbito \\
\hline 2 & S. viridans & IV & Óbito \\
\hline 3 & S. aureus & I & CFI \\
\hline 4 & S. aureus & IV & $\mathrm{CFI}$ \\
\hline 5 & Enterococcus & IV & CF I \\
\hline 6 & Enterococcus & II & CFI \\
\hline 7 & $(-)$ & IV & Óbito \\
\hline 8 & $(-)$ & IV & CFI \\
\hline 9 & S. viridans & II I & $\mathrm{CFI}$ \\
\hline 10 & S. epidermidis & II & CFI \\
\hline 11 & $(-)$ & II & CFI \\
\hline 12 & S. epidermidis & II I & Óbito \\
\hline 13 & S. viridans & II I & CFI \\
\hline 14 & $(-)$ & IV & CFII \\
\hline 15 & S. viridans & I I & CFI \\
\hline 16 & $(-)$ & IV & Óbito \\
\hline 17 & S. aureus & IV & Óbito \\
\hline 18 & $(-)$ & IV & Óbito \\
\hline 19 & S. aureus & IV & Óbito \\
\hline 20 & S. aureus & IV & Óbito \\
\hline 21 & S. aureus & IV & CFI \\
\hline 22 & Diphiteroidis & IV & CFIII \\
\hline 23 & S. aureus & II I & CFI \\
\hline 24 & $(-)$ & I I I & Óbito \\
\hline 25 & S. epidermidis & III & CFI \\
\hline 26 & $(-)$ & II & CFI \\
\hline 27 & $(-)$ & II I & Óbito \\
\hline 28 & $(-)$ & IV & CFI \\
\hline 29 & S . beta-haemoliticcus & II I & Óbito \\
\hline 30 & $(-)$ & IV & Óbito \\
\hline 31 & $(-)$ & II I & CFII \\
\hline 32 & S. viridans & I & CFI \\
\hline 33 & S. aureus & IV & Óbito \\
\hline \multicolumn{4}{|c|}{$\begin{array}{l}\text { S.aureus- Staphylococcus aureus; S.viridans- Streptococcus viridans; } \\
\text { S.epidermidis- Staphylococcus epidermidis; S.beta-haemoliticcus- } \\
\text { Streptococcus beta-haemoliticcus; (-) negativa. }\end{array}$} \\
\hline
\end{tabular}




\begin{tabular}{|c|c|c|c|}
\hline \multicolumn{4}{|c|}{$\begin{array}{l}\text { Tabela III - Análise multivariada (riscos proporcionais de cox). } \\
\text { Preditores de óbito intra-hospitalar a partir de dados obtidos na } \\
\text { admissão }\end{array}$} \\
\hline Variáveis & $\mathrm{H}-\mathrm{R}$ & I.C. & Valores de $\mathrm{p}$ \\
\hline Cardiopatia de base & 2,225 & $0,979-4,588$ & 0,051 \\
\hline Classe funcional & 3,474 & $1,469-8,216$ & 0,004 \\
\hline Leucometria & 5,062 & $1,304-19,652$ & 0,019 \\
\hline \multicolumn{4}{|l|}{ Vegetação ao } \\
\hline $\mathrm{ECO} 2-\mathrm{D}$ & 0,143 & $0,765-1,707$ & 0,513 \\
\hline
\end{tabular}

representou fator predisponente de óbito intra-hospitalar (dos 26 pacientes, $14(42,42 \%$ ) faleceram, enquanto que dos sete em CF I/II, todos evoluíram para cura $(\mathrm{p}=0,01))$. Entre os 17 casos que apresentaram complicações embólicas, 10 $(58,82 \%)$ faleceram, enquanto que entre os 16 em que não foram identificadas embolias, quatro $(25 \%)$ foram a óbito, sendo esta diferença marginalmente significativa $(\mathrm{p}=0,05)$.

No que se refere aos aspectos dos exames complementares e mortalidade, dos 13 pacientes em que a contagem global de leucócitos encontrava-se acima de 10.000/ $\mathrm{mm}^{3}$, nove $(69,23 \%)$ faleceram, contra cinco $(25 \%)$ dos 20 casos com leucócitos normais ( $\mathrm{p}=0,02)$. Não houve diferença significativa, quando analisados os resultados das hemoculturas (se positiva ou negativa) e a ocorrência de óbitos ( $\mathrm{p}>0,05)$, e, quando positiva, a relação entre os diversos agentes etiológicos e a mortalidade ( $\mathrm{p}>0,05)$. Também, não houve diferença entre as endocardites de origem estafilocócica e as por outros agentes ou com hemoculturas negativas quanto à indicação cirúrgica na fase ativa da doença.

Entre os que apresentaram vegetações ao ECO, nove (64\%) dos 14 casos de acometimento da valva mitral faleceram, contra três (33\%) dos nove casos de comprometimento da valva aórtica. Não houve diferença significativa, quando comparadas as valvas envolvidas e a mortalidade, nem quanto à presença ou ausência de vegetação e a mortalida$\operatorname{de}(\mathrm{p}>0,05)$.

Em relação à análise multivariada, foi construído modelo de riscos proporcionais de Cox com a CF, a leucometria, a cardiopatia de base e a presença ou não de vegetação ao ECO, com os dados da admissão. Apurou-se que, somente a CF na internação (hazard-ratio $=3,474$ com intervalo de confiança de $95 \%=1,469-8,216, p=0,004)$ e a leucometria $>10.000 / \mathrm{mm}^{3}$ (hazard-ratio=5,062 com intervalo de confian-

\begin{tabular}{|llcc|}
\hline \multicolumn{4}{|c|}{$\begin{array}{l}\text { Tabela IV - Análise multivariada (regressão logística múltipla). } \\
\text { Preditores de óbito intra-hospitalar a partir de dados obtidos na } \\
\text { admissão e durante a internação. }\end{array}$} \\
\hline Variáveis & O-R & IC & Valores de p \\
\hline Cardiopatia & 2,928 & $0,740-11,575$ & 0,125 \\
CF na admissão & 6,285 & $1,426-27,707$ & 0,015 \\
Embolia & 12,073 & $1,132-128,752$ & 0,039 \\
Leucometria & 1,257 & $0,146-10,839$ & 0,835 \\
\hline O-R- odds-ratio; IC- intervalo de confiança para 95\%. & \\
\hline
\end{tabular}

ça de 95\%=1,304-19,652), $\mathrm{p}=0,019)$, eram preditores independentes de óbito intra-hospitalar (tab. III).

A análise da sobrevida atuarial evidenciou que após 50 dias de internação, a sobrevida cumulativa dos pacientes em CF I/II foi de $90 \%$ e naqueles em CF III/IV de $68 \%$ $(\mathrm{p}=0,04)$ (fig. 1). Para o leucograma, os que apresentavam contagem de leucócitos $<10.000 / \mathrm{mm}^{3}$ na admissão tinham sobrevida cumulativa de $71 \%$ e os com valores maiores, de $29 \%$ ( $\mathrm{p}=0,03$ ), aos 61 dias de internação (fig. 2).

Com o objetivo de identificar os preditores independentes de óbito intra-hospitalar, a partir de dados obtidos na admissão e durante o período de internação, foi construído modelo de regressão logística múltipla por etapas com a cardiopatia de base, a CF e o leucograma na admissão, e a ocorrência de embolias durante a hospitalização. Foram preditores independentes de óbito intra-hospitalar a CF (odds-ratio $=6,285$ com intervalo de confiança de $95 \%=1,426-27,707, \mathrm{p}=0,015)$ e a ocorrência de embolias (odds-ratio $=12,073$ com intervalo de confiança de $95 \%=$ $1,132-128,752, \mathrm{p}=0,039)($ tab. IV).

\section{Discussão}

A EI permanece ainda, hoje, como condição prevalente de grande morbidade e taxa de mortalidade variando de $10 \mathrm{a}$ $36 \%$, a despeito do progresso de antibióticos de largo espectro, de novas técnicas cirúrgicas e de programas profiláticos ${ }^{10-14}$.

Tem sido observado que a faixa etária de maior percentual da doença tem migrado para as mais elevadas, atingindo nos EUA mais de 50\% dos pacientes acima dos 50 anos ${ }^{15,16}$, diferente do observado em nosso país, onde o maior percentual (53\%) situa-se na faixa etária de 11 a 30 $\operatorname{anos}^{5}$. A doença orovalvar reumática, seja de valva nativa ou de prótese, representou a maioria dos nossos casos $(75,75 \%)$, diferente dos resultados obtidos nos países desenvolvidos, onde representa de 10 a $25 \%{ }^{15,17}$, entretanto, semelhantes aos já relatados em outros de nossos centros ${ }^{5}$. As cardiopatias congênitas representaram $24 \%$ dos casos, e já predominam em pacientes com idades $<10$ anos, conforme relatado por Jorge e $\mathrm{col}^{18}$.

A valva mitral foi a mais comprometida (52\%), seguida do acometimento de ambas as valvas mitral e aórtica $(33 \%)$ e aórtica isolada em $14 \%$ dos casos. Entre as cardiopatias congênitas, a CIV foi a mais encontrada, em $37 \%$ dos casos, achados semelhantes aos descritos na população adulta ${ }^{19,20}$. As possíveis portas de entrada relatadas em $24 \%$ dos casos são as mesmas descritas na literatura: manipulação dentária, traumatismos com infecção de partes moles, entre outras. Neste estudo não detectamos uso de drogas ilícitas injetáveis, nem portadores da síndrome de imunodeficiência adquirida, talvez devido à baixa faixa etária da população, já que a maioria das séries relata esta ocorrência em idades entre 25 e 30 anos $^{21}$. Vale a pena salientar que todos os pacientes apresentavam condição cardíaca predisponente e os que apresentavam EI no lado direito do coração eram portadores de cardiopatia com comunicação es- 
querda-direita. As características deste grupo estudado são decorrentes do tipo de trabalho desenvolvido no NESA, com programas específicos de atendimento a adolescentes portadores de cardiopatia, incluindo cirurgia cardíaca, servindo como centro de referência estadual. Portanto, todos os pacientes internados com diagnóstico de EI, já estavam inseridos em algum programa de atendimento ou foram transferidos de hospitais da rede pública para tratamento específico ou correção de complicações. Assim, $78,78 \%$ achavam-se em CF III/IV, condição que representou fator preditor independente de mortalidade altamente significativo, situando-se em $42,43 \%$ na fase intra-hospitalar, maior do que os encontrados em outros centros ${ }^{1,5}$. Apesar de não apresentar significado estatístico, provavelmente pelo pequeno número da amostra analisada, o percentual expressivo de endocardites estafilocócicas $(42,85 \%)$ pode ter contribuído para a alta taxa de mortalidade.

Embolias foram detectadas em 51,51\% dos pacientes, sendo que em $41,17 \%$ para SNC. Nem sempre as complicações embólicas, principalmente as neurológicas, foram clinicamente evidentes. Somente o alto grau de suspeição vigente na instituição, permitiu-nos a realização de exames que comprovaram a suspeita clínica, já que, conforme relatado por vários autores e confirmado neste estudo, complicações embólicas representam fator preditor de mortalidade intra-hospitalar ${ }^{5,12,22}$.

Em relação aos exames complementares, observamos leucocitose em $39,39 \%$ e sua relação significativa com a mortalidade, aspecto que é pouco descrito na literatura, exceto nos casos de instalação aguda da doença, em particular, nas endocardites estafilocócicas ${ }^{23,24}$. O maior percentual de quadros graves (CF III/IV) e de infecções estafilocócicas observado, pode representar as possíveis explicações para este achado. O agente etiológico mais isolado foi o Staphylococcus aureus em 42,85\% dos casos, seguido do Streptococcus, grupo viridans, em $21 \%$. Valores que são diferentes dos descritos em outros centros ${ }^{12,25-26}$, mostrando ainda a predominância da endocardite estreptocócica sobre a estafilocócica, mesmo na população adulta de nossa instituição ${ }^{27}$. Apesar da maioria dos autores relatar maior percentual de mortalidade nas endocardites estafilocócicas e maior gravidade da doença quando causada por esse agente ${ }^{5,17,26}$, em população de adolescentes, não observamos diferença significativa entre os diversos agentes etiológicos (ou hemoculturas negativas) e a mortalidade ou gravidade da doença. Tais fatos se devem, possivelmente, ao pequeno número da nossa casuística que, apesar de expressivo no que diz respeito à doença, é pequeno, no entanto, para análise estatística.

OECO foi de grande importância para a elucidação do diagnóstico e, também, para o acompanhamento dos pacientes. Observamos imagens compatíveis com vegetação em $75,75 \%$, percentual próximo ao referido na literatura, que se situa em torno de $80 \%{ }^{28,29}$. A pouca idade dos pacientes que proporciona a obtenção de boas imagens, bem como o pequeno número com próteses cardíacas justifica o percentual encontrado, já que no maior período de observação deste estudo, a instituição não dispunha de sondas transesofágicas. Semelhante ao descrito ${ }^{1,16,30}$, o maior percentual de vegetações foi detectado na valva mitral (56\%), seguido da valva aórtica (36\%), sendo, na maioria dos casos, a doença reumática a condição predisponente, refletindo a elevada freqüência das lesões reumáticas crônicas em nosso meio. Vegetações nas valvas tricúspide e pulmonar foram detectadas em $8 \%$, em decorrência do baixo número de cardiopatias congênitas na nossa série e pela ausência de usuários de drogas intravenosas. Alguns autores ${ }^{29,31}$ atribuem valor prognóstico no que diz respeito à mortalidade $\mathrm{e}$ indicação cirúrgica e à presença/tamanho da vegetação ao ECO. Tais fatos ainda permanecem controversos e não foram confirmados na nossa série, igualmente descritos por Mansur e col ${ }^{5}$.

Em relação ao tipo de tratamento, $19(57,58 \%)$ pacientes foram submetidos exclusivamente a tratamento clínico, sendo que $9(47 \%)$ faleceram, enquanto $14(43 \%)$ foram submetidos a tratamento clínico e cirúrgico e $5(35 \%)$ faleceram. A principal causa de mortalidade, em ambos os grupos, foi a insuficiência cardíaca congestiva, que representa segundo dados da literatura, a principal e mais temida complicação da doença $^{1,5,22,32}$. Não observamos diferença estatística significativa entre a mortalidade e o tratamento empregado, possivelmente, pelo pequeno número da amostra e, também, pelo período de tempo de observação, sendo que os dados iniciais foram obtidos na década de 80, quando ainda havia certa relutância na indicação cirúrgica na fase ativa da doença.

A orientação na escolha da prótese, a ser implantada, tem sido a prótese mecânica, apesar de relatos de maior risco de "repetição" da doença, quando comparada à biológi$\mathrm{ca}^{5}$, o que não foi confirmado no nosso grupo, já que apenas um dos pacientes apresentou novo episódio 12 meses após a cirurgia e, além do mais, implante de próteses biológicas em pacientes de baixa idade pode acarretar disfunção precoce da prótese, com necessidade de nova intervenção cirúrgica a curto prazo e, conseqüentemente, aumento da morbimortalidade, dados comprovados em trabalho sobre a durabilidade de próteses cardíacas em adolescentes ${ }^{33}$.

Apesar do percentual expressivo de casos de EI em nossa instituição, alguns resultados podem não representar as características da doença na população estudada, já que pelas características da instituição, esses casos são previamente selecionados, quer pela gravidade, quer pelo alto percentual de cirurgias cardíacas realizadas no hospital. No entanto, o valor numérico, 33, não é, do ponto de vista estatístico, suficiente para análises mais amplas. A ampliação deste estudo, com aumento do número de casos, se faz necessária, para melhor avaliação da doença nesta faixa etária.

No entanto, podemos concluir que a EI na adolescência assemelha-se em alguns aspectos à EI do adulto, tendo a cardiopatia reumática como o principal fator predisponente e, em outros, à EI da infância, tendo o S.aureus como o agente etiológico mais comumente encontrado. A mortalidade da doença é elevada e representa como preditores de mortalidade intra-hospitalar, a CF III/IV na admissão, a ocorrência de fenômenos embólicos e a leucocitose. 


\section{Referências}

1. Von Reyn CF, Levy BS - Infective endocarditis: na analysis based on strict case definitions. Ann Intern Med. 1981; 94:505-17.

2. Kaye D - Definitions and demographic characteristics. In: Kaye D - Infective Endocarditis. Baltimore: University Park Press, 1976: 1-10.

3. Johnson CM, Rhoddes KH - Pedriatric endocarditis. Mayo Clin Proc 1982; 57 : 86-94.

4. Choudhury R, Grover A, Varma J et al - Active infective endocarditis observed in a Indian hospital 1981-1991. Am J Cardiol 1992; 70: 1453-8.

5. Mansur AJ, Grinberg M, Gallucci SDD et al - Endocardite infecciosa. Análise de 300 episódios. Arq Bras Cardiol 1990; 54: 13-21.

6. Watanakunakorn C, Burket T - Infective endocarditis at a large community teaching hospital, 1980-1990. A review of 210 epidodes. Medicine 1993; 72: 90 102.

7. Lowes JA, Williams G, Tabaqchali S et al - 10 years of infective endocarditis at St Bartholomew's hospital: analysis of clinical features and treatment in relation to prognosis and mortality. Lancet 1989; 1: 133-6.

8. Faiman L, Prince A, Gersony WM - Pedriatric infective endocarditis in modern era. J Pedriatr 1993; 122: 847-53.

9. Durack Dt, Lukes AS, Bright Dk et al - New criteria for diagnosis of infective endocarditis: utilization of specific echocardiographic findings. Am J Med 1994 96: 200-9.

10. Korzenioski O, kaye D - Infective Endocarditis. In: Braunwald E - Heart Disease: A Textbook of Cardiovascular Medicine. $4^{\text {th }}$ ed. Philadelphia: WB Saunders, 1992: 1078.

11. Goodwin JF- The challenge and the reproach of infective endocarditis. Br Heart J 1985; 54: 115-8.

12. Mansur AJ, Grinberg M, Belloti G, Jatene A, Pileggi F- Infective endocarditis in the 1980 experience at a heart hospital. Clin Cardiol 1990; 13: 623-30.

13. Alsip Sg, Blackstone EH, Kirklin JW, Cobbs CG-Indications for cardiac surgery in patients with active infective endocarditis. Am J Med 1985; 70: 138-49.

14. Aslamaci S, Dimitri WR, Williams BT - Operative considerations in the active native valve endocarditis. J Cardiovascular Surg 1989; 30: 328-33.

15. McKinsey Ds, Ratts TE, Bisno AL - Underlying cardiac lesions in adults with infective endocarditis. The changing spectrum. Am J Med 1989; 82: 681-8.

16. Weinberger J, Rotenberg Z, Zacharovitch $D$ et al - Native valve endocarditis in the 1970 's versus the 1980 's: underlying cardiac lesions and infecting organisms. Clin Cardiol 1990; 13; 94-8.

17. Kaye D-Changing patterns of infective endocarditis. Am J Med 1985(suppl 6B); 78: $157-62$
18. Jorge SC, Caixeta AM, Abizaid A et al - Endocardite infecciosa na infância e adolescência. Arq Bras Cardiol. 1994; 63: 173-7.

19. Gersony WM, Hayes CJ, Driscoll DJ et al - Bacterial endocarditis in patients with aortic stenosis, pulmonary stenosis or ventricular septal defect. Circulation 1993; 87(suppl I): 1-121.

20. Moller JH, Anderson RC - 1000 consecutive children with a cardiac malformation with 26-to-36 year follow-up. Am J Cardiol 1992; 70: 661-7.

21. Gringerg M, Mansur AJ, Uip DE - Infecção por HIV. Um novo aspecto a ser considerado na endocardite infecciosa. Arq Bras Cardiol 1992; 59: 347-50.

22. Garvey GJ, New HC - Infective endocarditis - a review of endocarditis at the Columbic Presbiterian Medical Center 1968-1973. Medicine 1978; 57: 105-127.

23. Franken RA - Exames de laboratório no diagnóstico e tratamento da endocardite infecciosa. Rev Soc Cardiol ESP 1995; 4: 398-401.

24. Woo KS, Lam YM, Kwosk HT, Tse LK, Vallence-Owen J - Prognostic index in prediction of mortality from infective endocarditis. Int J Cardiol 1989; 24: 47-54.

25. Iga K, Hori K, Matsumara T, Tomonaga Get al-Native valve infective endocarditis in adults-analysis of 32 consecutive cases over a tem period from 1980 to 1989. Jpn Circ J 1991; 55: 437-42.

26. Thamlikitkul V, Praditsuwan R, Permpikul C, Jootan D - Native valve endocarditis at Siriraj hospital. J Med Assoc Thai 1991; 74: 313-22

27. Oliveira R - Aspectos epidemiológicos da endocardite infecciosa em hospital geral no período 1982-1986. Rio de Janeiro (Tese de Mestrado). Centro Biomédico da UERJ, 1989

28. Assef JE, Pontes JR, Ginenez VMet al - Endocardite infecciosa. Estudo Dopplerecocardiográfico prospectivo. Arq Bras Cardiol 1990; 55: 19-25.

29. Mugge A, Daniel WG, Frank G, Lichten PR - Echocardiography in infective endocarditis: reassessment of prognostic implications of vegetation size determined by the transthoracic and transesophageal aproach. J Am Coll Cardiol 1989; 14: 631-8.

30. Terpenning MS, Buggy BP, Kauffman CA - Infective endocarditis: clinical features in young and elderly patients. Am J Med 1987; 83: 626-34

31. Sutton MS, Lee RT - Diagnosis and medical management of infective endocarditis: Transthoracic and transesophageal echocardiography. J Card Surg 1990; 5 : 39-43.

32. Braudenburg RO, Guliani E, Wilson WR, Geraci JE-Infective endocarditis. A 25 years overview of diagnosis and therapy. J Am Coll Cardiol 1983; 280-97.

33. Fernandes LA - Avaliação das próteses valvares em criança e adolescentes: evolução clínica eletrocardiográfica radiológica e ecocardiográfica (Tese de Mestrado). Rio de Janeiro: UERJ, 1991. 